\title{
Mathematical Modelling of Two-axis Photovoltaic System with Improved Efficiency
}

\author{
Fevzi Kentli ${ }^{1}$, Musa Yilmaz ${ }^{2}$ \\ ${ }^{I}$ Department of Mechatronics Engineering, Technology Faculty, Marmara University, \\ Istanbul, Turkey \\ ${ }^{2}$ Department of Electronics and Communications Engineering, Technology Faculty, \\ Batman University, Batman, Turkey \\ fkentli@marmara.edu.tr
}

\begin{abstract}
Design of a two axis sun tracking system is much more complex compared to one axis. Tracking the sun could be accomplished by either running algorithms integrated to system or using photoresistances. In this study, mathematical formulation of sun tracking system using photoresistances and its mechanical components, is derived. A new sun tracking system is proposed considering the results of calculation. The system capable of moving in two axis tracks the sun from sunrise to sunset as sunlight is always normal to its surface. So, it profited from sunlight optimally. System stops during cloudy weather or after sunset and so inessential power consumption is prevented. Moreover, consumed energy by the motors providing movement of system is minimized by the new system. Designed two axis sun tracking system is compared with a fixed system and it is observed that $30 \%$ more electrical energy is produced annually.
\end{abstract}

Index Terms-Two axis tracking, solar energy, sun tracking.

\section{INTRODUCTION}

The best photovoltaic panels are made from silicon (Si) material which are having more than $24 \%$ efficiency by the technological developments during the last 30 years. They are known as high efficient single crystal panels. As they are expensive, technology on thin film panels has improved in last 20 years. In the meantime, production of photovoltaic panels has increased annually approximately $30 \%$ around the world [1].

Optimal utilization of photovoltaic panels under different weather conditions is one of the economic problems. To use in rural area or away from city center is much more convenient for these cases. Photovoltaic panels could be uneconomical since lack of sunlight or severe weather conditions. That is why so many studies on declination angle have been done to maximize efficiency [2]-[4].

Output power of any photovoltaic system depends on obtained solar energy. To provide more solar energy as an input to system, it is required to track the sun. Applications brought out different sun tracking systems. In this study, a photovoltaic two-axis sun tracking system is used. Evaluation of different systems is based on annual

Manuscript received December 14, 2014; accepted March 24, 2015.

This research was funded by a grant from the Research Council of Scientific Research Projects Coordinator-Dicle Uni. (12-MYO-146) and Marmara Uni.(FEN-D-130515-0204). measurement of sunlight as input of system and power as output of system [1].

Efficiency of photovoltaic panels reaches maximum when sunlight comes normal to surface. So, to control the motors in suitable moments is needed to provide this condition. Control circuit achieves by the help of photoresistances. Control circuit consists of electronic and mechanical systems as shown in Fig. 1. Providing sunlight always normal is accomplished by calculating sun movement and using 5 LDR in control circuit. 2 LDR is used for detecting horizontal axis (East-West) and 2 LDR is used for detecting vertical (North-South) and one LDR is used to check the sun. So, motors can always track sun as sunlight is always normal to surface [4]-[8].

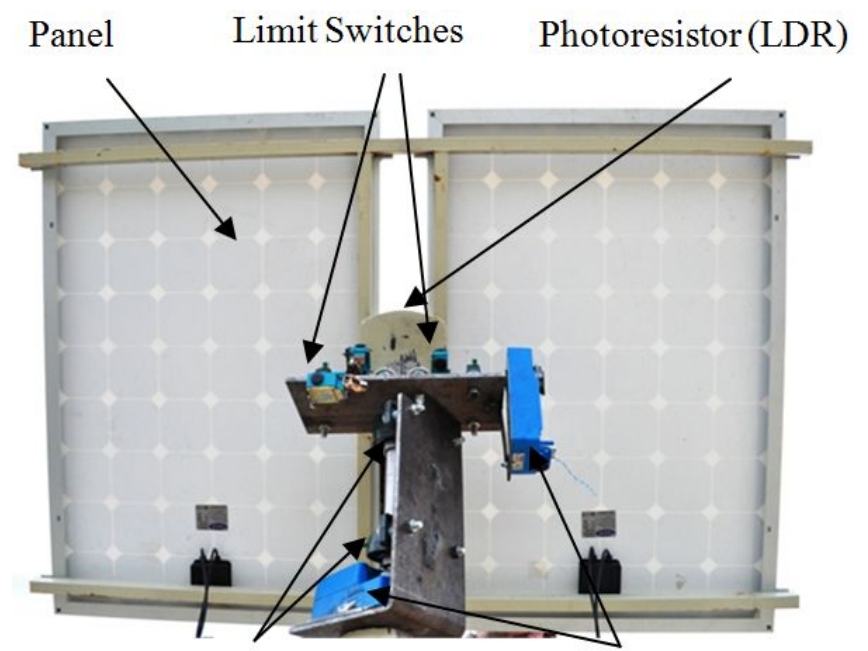

Roller Bearings DamperMotor

Fig. 1. Improved two-axis sun tracking system.

Design of two-axis photovoltaic panel (solar tracking) system is a difficult task. Efficiency and cost of tracking system should be optimized. Energy consumption is concerned in tracking system, differently than fixed ones. This is a factor affecting efficiency of system. To minimize this effect, literature is investigated and a DC motor having gear system (Damper Motor) with low energy consumption is found as best choice [1].

\section{Modelling OF Mechanic Systems}

In this section, performed two-axis photovoltaic panel 
(sun tracking) system will be addressed. In tracking systems, consumption of energy is in question differing than fixed systems. It affects the system efficiency. To minimize this effect, literature is investigated and a DC motor with gear unit which has very low energy consumption is selected. Both motors have same properties. Figure 2 shows the tracking system as mounted to the roof of building.

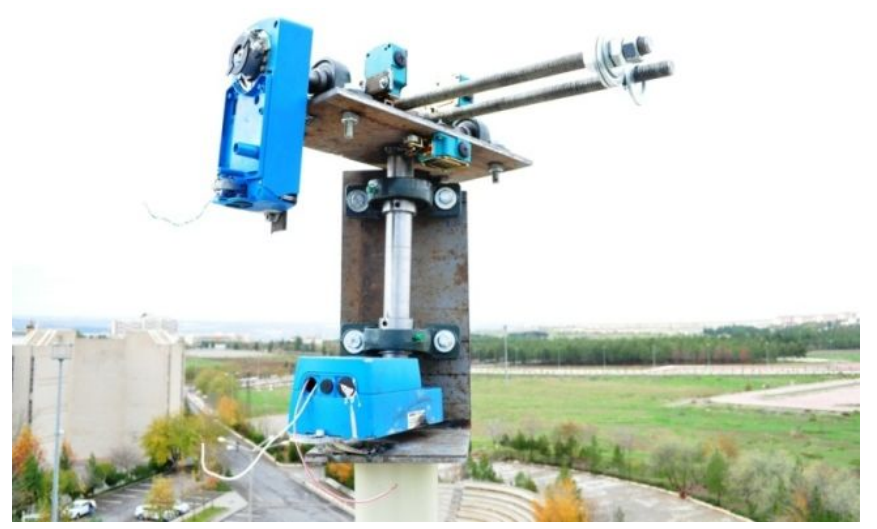

Fig. 2. Picture of the used two-axis tracking system.

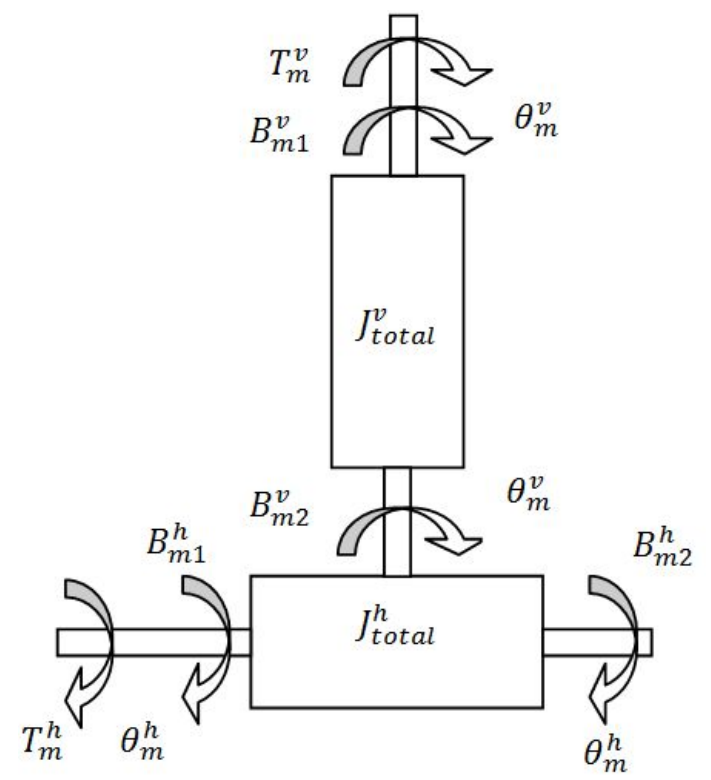

Fig. 3. Mechanical model of two-axis tracking system.

In modelling the system, (1)-(5) (derived for system shown in Fig. 4) are used for horizontal (East-West) and vertical (North-South) axis [4].

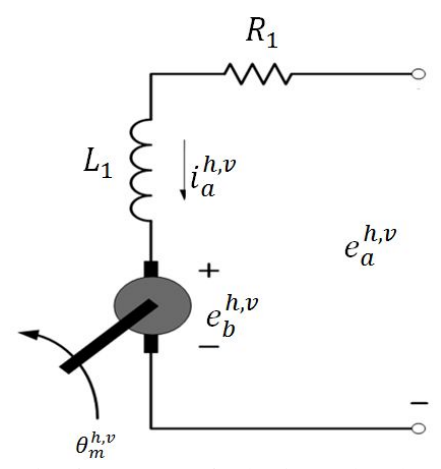

Fig. 4. Electric circuit of DC motor for horizontal and vertical axis.

This tracking system makes movement in two-axis. Horizontal (East-West) and vertical (North-South) axis are moved separately even they are connected together. System is moving in horizontal and vertical directions independently as shown in Fig. 2 and Fig. 3. Both systems are facilitated by using two roller bearings. Wind load data for 10 years are taken from meteorology for the place where experiments are done and it is seen that they can be neglected because their values are very low $(<3.5 \mathrm{~m} / \mathrm{s})$. But system is built as withstanding the loads over average [4].

For vertical (North-South) axis of the system:

$$
\begin{gathered}
e_{a}^{\text {vertical }}=i_{a}^{v} R_{1}+L_{1} \frac{d i_{a}^{v}}{d t}+e_{b}^{v} \\
e_{b}^{\text {vertical }}=K_{b}^{v} \frac{d \theta_{m}^{v}}{d t}
\end{gathered}
$$

where $K_{T}^{v}$ - torque constant, $K_{b}^{v}$ - electromotive force constant.

$$
e_{a}^{v}=i_{a}^{v} R_{1}+L_{1} \frac{d i_{a}^{v}}{d t}+K_{b}^{v} \frac{d \theta_{m}^{v}}{d t}
$$

Mathematical modelling of vertical axis of mechanical systems is (Fig. 3.).

$$
\begin{gathered}
B_{t}^{v}=B_{m 1}^{v}+B_{m 2}^{v}-\text { viscous damper coefficient of bearing. } \\
T_{m}^{v}=J_{t}^{v} \frac{d^{2} \theta_{m}^{2}}{d t^{2}}+B_{t}^{v} \frac{d \theta_{m}^{v}}{d t} \\
T_{m}^{v}=K_{T}^{v} \cdot i_{a}^{v}
\end{gathered}
$$

where $J_{t}^{v}=1$ - equivalent moment of inertia for torque of motors $\left[\mathrm{kg} \cdot \mathrm{mm}^{2}\right]$.

$$
K_{T}^{v} \cdot l_{a}^{v}=J_{t}^{v} \frac{d^{2} \theta_{m}^{2}}{d t^{2}}+B_{t}^{v} \frac{d \theta_{m}^{v}}{d t} .
$$

Laplace transform of equation:

$$
\begin{gathered}
E_{a}^{v}(s)=I_{a}^{v}(s) R_{1}+L_{1} s I_{a}^{v}(s)+K_{b}^{v} s \theta_{m}^{v}(s), \\
K_{T}^{v} I_{a}^{v}(s)=J_{t}^{v} s^{2} \theta_{m}^{v}+B_{t}^{v} s \theta_{m}^{v}(s) .
\end{gathered}
$$

Equation (7) is shortened as

$$
I_{a}^{v}(s)=\frac{E_{a}^{v}(s)-K_{b}^{v} s \theta_{m}^{v}(s)}{R_{1}+L_{1} s}
$$

When (9) is substituted into (8):

$$
\begin{gathered}
K_{T}^{v}\left(\frac{E_{a}^{v}(s)-K_{b}^{v} s \theta_{m}^{v}(s)}{R_{1}+L_{1} s}\right)=J_{t}^{v} s^{2} \theta_{m}^{v}(s)+B_{t}^{v} s \theta_{m}^{v}(s), \\
K_{T}^{v} E_{a}^{v}(s)-K_{T}^{v} K_{b}^{v} s \theta_{m}^{v}(s)= \\
=\left[J_{t}^{v} s^{2} \theta_{m}^{v}(s)+B_{t}^{v} s \theta_{m}^{v}(s)\right]\left(R_{1}+L_{1} s\right), \\
K_{T}^{v} E_{a}^{v}(s)=J_{t}^{v} s^{2} \theta_{m}^{v}(s) R_{1}+J_{t}^{v} s^{3} L_{1} \theta_{m}^{v}(s)+ \\
+B_{t}^{v} s R_{1} \theta_{m}^{v}(s)+B_{t}^{v} s^{2} L_{1} \theta_{m}^{v}(s)+K_{T}^{v} K_{b}^{v} s \theta_{m}^{v}(s),
\end{gathered}
$$




$$
\begin{gathered}
K_{T}^{v} E_{a}^{v}(s)=\theta_{m}^{v}(s)\left[J_{t}^{v} s^{3} L_{1}+\right. \\
\left.+\left(J_{t}^{v} R_{1}+B_{t}^{v} L_{1}\right) s^{2}+\left(B_{t}^{v} R_{1}+K_{T}^{v} K_{b}^{v}\right) s\right], \\
\frac{\theta_{m}^{v}(s)}{E_{a}^{v}(s)}= \\
=\frac{K_{T}^{v}}{J_{t}^{v} L_{1} s^{3}\left(J_{t}^{v} R_{1}+B_{t}^{v} L_{1}\right) s^{2}+\left(B_{t}^{v} R_{1}+K_{T}^{v} K_{b}^{v}\right) s} .
\end{gathered}
$$

It is not needed to derive the equations for horizontal axis because both axis are under same conditions [3], [4].

\section{MODELling OF SUN TRACKING}

Modelling for one axis is enough as system works same at both axes. Working principle of system is shown in Fig. 6. 4(four) photoresistances (LDR) placed onto panel surface to direct to correct $\alpha$ angle (angle of incidence). LDRs are placed so that every LDR has same amount of sunlight when sunlight comes as normal to surface. LDRs are used as current source and are connected to different channels of operative amplifier. As currents of LDRs are related to range of sunlight, an error occurs when sun light doesn't come to normal to surface. Motor arrange system back to normal by applying this voltage difference to servo amplifier.

\section{A. Coordinate System}

Centre of coordinate system is assumed to be on output gear. When reference axis is accepted as the fixed body of DC motor, all rotations are measured around this axis. Sun axis (line connecting centre of output gear with sun) states $\theta_{r}(t)$ reference angle (Fig. 5). Purpose of control system is to minimize the angle difference, $\alpha(t)$, between $\theta_{r}(t)$ and $\theta_{0}(t)$ where $\theta_{0}(t)$ represents the angle between reference axis and tracking axis.

$$
\alpha(t)=\theta_{r}(t)-\theta_{0}(t)
$$

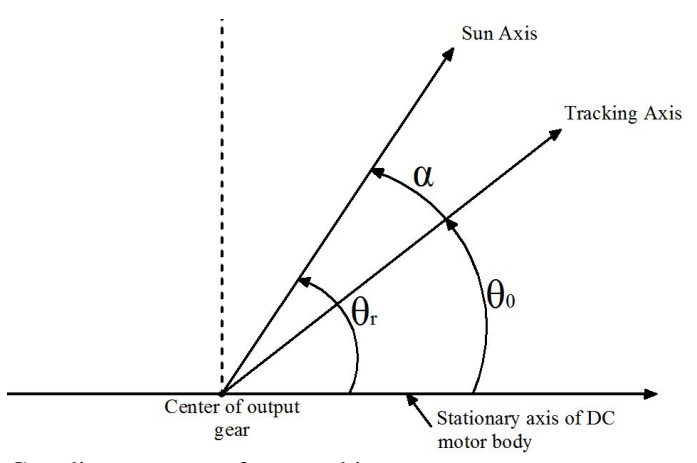

Fig. 5. Coordinate system of sun tracking system.

\section{B. Error Detector}

When tracking axis overlaps with sun axis: $\alpha(t)=0$ and $i_{a}(t)=i_{b}(t)=I$ or $i_{a}(t)-i_{b}(t)=0$. As shown in Fig. 6, sunlight having $\mathrm{W}$ width reaches at certain $\alpha$ angle to A cell with $o a$ width and to B cell with $o b$ width:

$$
o a=\frac{W}{2} L \tan \alpha(t)
$$

$$
o b=\frac{w}{2}-L \tan \alpha(t)
$$

On the other hand, when $2 I$ is the maximum current of a LDR and $0 \leq \tan \alpha(t) \leq W / 2 L$ as $i_{a}(t)$ current is related with $o a$ and $i_{b}(t)$ current is related with $o b$ :

$$
\begin{aligned}
& i_{a}(t)=I+\frac{2 L I}{W}+\tan \alpha(t), \\
& i_{b}(t)=I-\frac{2 L I}{W}+\tan \alpha(t) .
\end{aligned}
$$

Sunlight goes only onto A cell (LDR1) and $i_{a}(t)=2 I, i_{b}(t)=0$ when $W / 2 L \leq \tan \alpha(t) \leq(C-W / 2) /$ $/ L$. When $(C-W / 2) L \leq \tan \alpha(t) \leq(C+W / 2) / L, i_{a}(t)$ current goes through zero from 2I. When $\tan \alpha(t) \geq(C+W / 2) / L, \quad i_{a}(t)-i_{b}(t)=0 . \quad$ Nonlinear characteristic shown in Fig. 7 can be used to define error detector. As $\alpha(t)$ angle is very small, $\alpha(t)$ is used instead of absis $\tan \alpha(t)$.

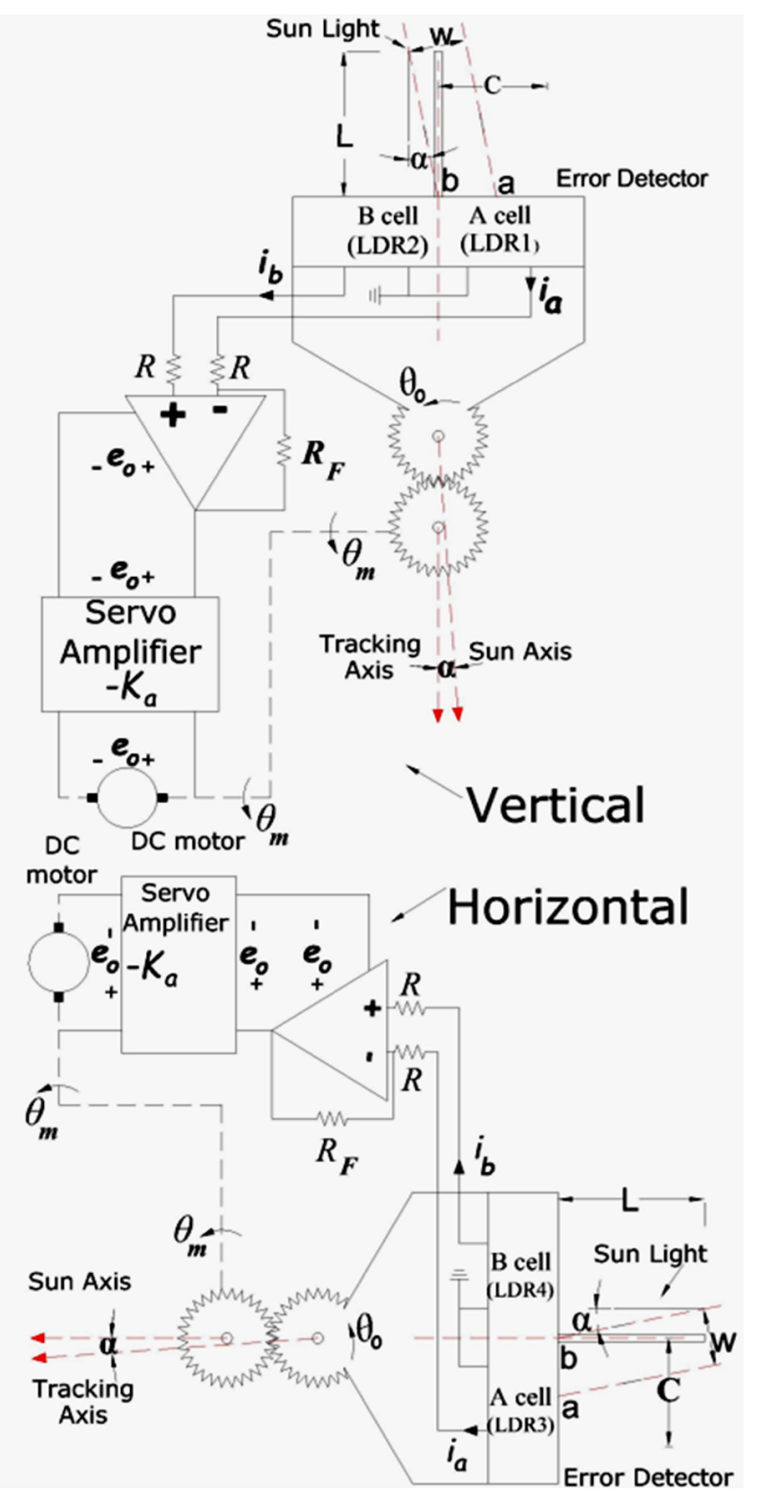

Fig. 6. Working principle scheme of improved two-axis solar tracking system [4]. 


\section{Operative Amplifier}

There is following relation between $i_{a}(t)$ and $i_{b}(t)$ and operative amplifier

$$
e_{o}(t)=-R_{F}\left[i_{a}(t)-i_{b}(t)\right]
$$

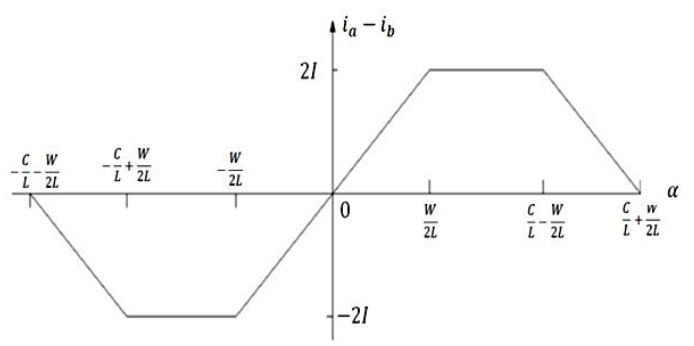

Fig. 7. Nonlinear characteristic of error detector.

\section{Servo Amplifier}

Gain of servo amplifier is $-K$. Output of servo amplifier as shown in Fig. 6 could be written as

$$
e_{a}(t)=-K e_{o}(t)
$$

\section{E. Damper Motor}

Damper motor consists of two components. First is gear unit and second is DC motor. Angular position $\theta_{o}$ of output gear is related with angular position of motor $\theta_{m}$ and $1 / \mathrm{n}$ gear ratio

$$
\theta_{o}=\frac{1}{n} \theta_{m}
$$

While $\mathrm{J}$ and $\mathrm{B}$ represents inertia and friction coefficient of motor shaft of DC motor:

$$
\begin{gathered}
e_{a}(t)=R_{a} i_{a}(t)+e_{b}(t), \\
e_{b}(t)=K_{b} \omega_{m}(t), \\
T_{m}(t)=K_{i} i_{a}(t), \\
T_{m}(t)=J \frac{d \omega_{m}(t)}{d t}+B \omega_{m}(t) .
\end{gathered}
$$

Block diagram showing functional relations of system is given in Fig. 8.

It is not needed to derive equations for vertical axis as both axes are under same conditions [4], [9].

\section{CONCLUSIONS}

The system whose mathematical model is derived is working properly. Sunlight is provided as always normal to panel surface at vertical or horizontal axis because it tracks the sun in both axes. Movement in one axis brings a shift in other axis as sun moves in an elliptical path. But, how much is shifted is calculated by the LDRs and system is corrected to provide sunlight as always normal to surface. Designed system is compared with a fixed one shown in Fig. 9. Designed two axis sun tracking system is producing $30 \%$ more energy annually as shown in Fig. 10 [10]. Consumed energy by mechanical system during tracking the sun like friction losses has such a negligible value that it is omitted.

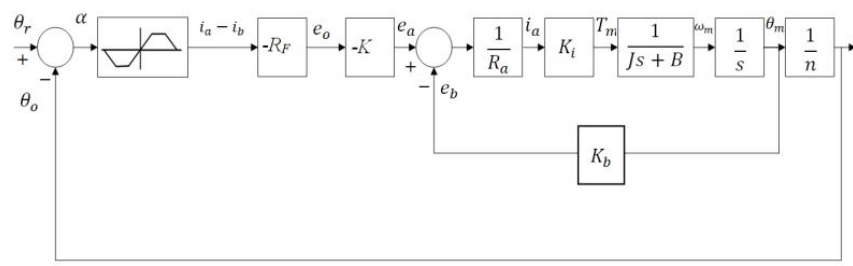

Fig. 8. Block diagram of sun tracking system [4].

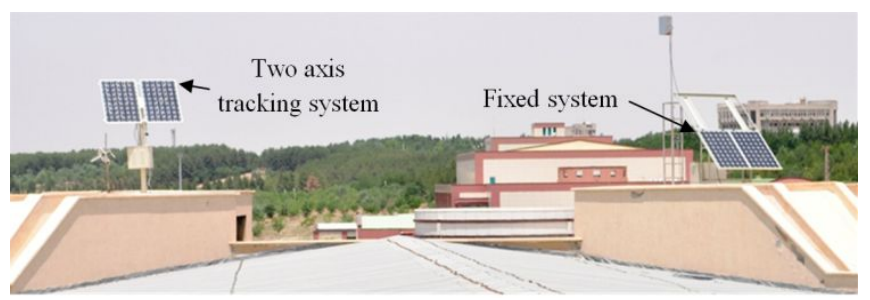

Fig. 9. View from the rooftop systems.

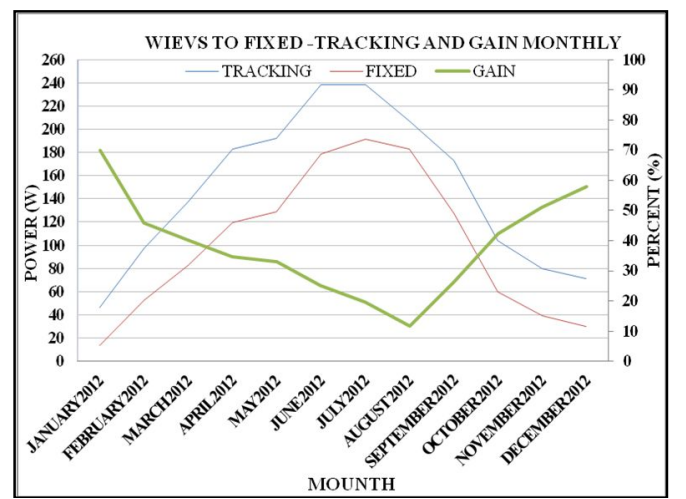

Fig. 10. Experimental average monthly total gain (\%).

It is recommended to researchers to focus on comparing this system with other sun tracking systems and with the ones using integrated algorithms for further studies.

\section{REFERENCES}

[1] F. Kentli, M. Yilmaz, "Obtaining the optimum efficiency electrical energy under Diyarbakır conditions using solar tracking system involving PV panel", EEST Part A, pp. 613-620, 2012.

[2] J. Samimi, E. A. Soleimani, M. S. Zabihi, "Optimal sizing of photovoltaic systems in varied climates", Elsevier. Solar Energy, vol. 60, no. 2, pp. 97-107, 1997. [Online]. Available: http://dx.doi.org/ 10.1016/S0038-092X(96)00165-X

[3] A. Senpinar, M. Cebeci, "Evaluation of power output for fixed and two-axis tracking PVarrays", Applied Energy, vol. 92, pp. 677-685, 2012. [Online]. Available: http://dx.doi.org/10.1016/j.apenergy. 2011.07.043

[4] C. B. Kho, "Otomatik kontrol sistemleri", Literatur Yayincilik, pp. 207-209, Istanbul, 2002. [in Turkish]

[5] S. Jacenas, "An algorithm to estimate thermal radiance centre in the sky hemisphere for automotive solar sensor validation", Elektronika Ir Elektrotechnika, vol. 6, pp. 73-76, 2011. [Online]. Available: http://dx.doi.org/10.5755/j01.eee.112.6.449

[6] D. Vasarevicius, R. Martavicius, "Solar irradiance model for solar electric panels and solar thermal collectors in Lithuania", Elektronika Ir Elektrotechnika, vol. 2, pp. 3-6, 2011. [Online]. Available: http://dx.doi.org/10.5755/j01.eee.108.2.132

[7] D. Lauria, M. Coppola, "Design and control of an advanced PV inverter", Solar Energy, vol. 110, pp. 533-542, 2014. [Online]. Available: http://dx.doi.org/10.1016/j.solener.2014.09.040

[8] K. Kachhiya, M. Lokhande, M. Patel, "Matlab/simulink model of solar PV module and MPPT algorithm", in Proc. National Conf. on Recent Trends in Engineering and Technology, 2011.

[9] E. K. Akpinar, F. Kocyigit, "Energy and exergy analysis of a new flat-plate solar air heater having different obstacles on absorber plates", Applied Energy, vol. 87, no. 11, pp. 3438-3450, 2010. [Online]. Available: http://dx.doi.org/10.1016/j.apenergy.2010.05.017

[10] M. Yilmaz, F. Kentli, "Increasing of electrical energy with solar tracking system at the region which has Turkey's the most solar energy potential", JOCET, vol. 3, no. 4, pp. 287-290, 2015. 